\title{
Service Discovery Integrated Network Platform
}

\author{
Kai-Oliver Detken, Ioannis Fikouras and Panos Phillipopoulos
}

Telscom AG, Sanrainstr. 17, 3007 Bern, Switzerland, BIBA, Hochschulring 20, 28359 Bremen, Germany, OTE Consulting S.A., 32, Kifissias Ave., GR-15125 Maroussi, Athens, Greece

\begin{abstract}
The ever increasing number of nomadic users will create a trend towards networking technologies that support mobility. Although wireless networks have already become commonplace and continuously expand their area of application with new technologies, like Bluetooth, wire-line networks as backbones or broadband bearer technologies will continue to play a significant role. A scenarios where the user has the requirement of using all available services on the road just as he would at home, in other words without needing to know any details about the network infrastructure - whether it will be by plugging your laptop to hotel network or turning on your wireless LAN on your PDA - will become more and more common. It is in situations like these where agent technologies will become very useful. User agents on user's device can discover the network, and locate and communicate with services on the network transparently to the user.
\end{abstract}

Key words: Seamless communication, mobility, service discovery, integrated network plattform

\section{INTRODUCTION}

Significant technological advances are taking place in recent years in the areas of palm-sized computers and wireless communications, accompanied by an infiltration of the Internet in all aspects of our lives. Consequently, technologies that allow the integration of existing and foreseen heterogeneous and homogenous networks into a single platform capable of supporting seamless user roaming between them will be of major importance. Nevertheless, the rapid growth of the Internet user base has not 
been accompanied by an equivalent evolution in the corresponding products and services for mobile customers. Existing systems do not maintain the capacity to adapt to changes in the user's location and preferences. It is understood that such services can only be realised through assistance of advanced positioning mechanisms determining the physical location of the user. However, the evaluation of this information is rarely enough for the resolution of service discovery services, mostly because there is no direct correspondence between the virtual (Internet) and physical (real-world) space.

The concepts addressing these issues presented in this paper have been implemented and tested in the SMONET-NGNI ${ }^{1}$ demonstrator. The demonstration software is not a simulator. It is a fully functional system operating within specified bounds.

\section{INTEGRATED NETWORK PLATFORM}

The lack of considerations for mobility management in the original Internet Protocol design indicate that a mobile node would be reachable only as long as it remained within the boundaries of a given IP administrative domain (IP network). Should a mobile node change its point of attachment to another IP network, it would remain unreachable by all communication peers until it returned to its home environment. This significant restriction forces users to remain under the influence of a single service provider, or network technology (i.e. GSM), in spite of utilising mobile, or portable devices. However, the availability of wireless and wire-line communication media will continue to increase accompanied by a plethora of access devices. In addition, the turn of operators towards license-free frequencies [1] and their eventual congestion will lead to the realisation of alternative dynamics based on the common convention that users may utilise one another's resources to mutually form a dynamic network structure. This solution poses as a lowcost, high-complexity alternative to conventional systems, one that will dictate a significant shift in complexity from the network towards the enddevices. This will give rise to a new generation of user equipment with several access interfaces allowing simultaneous connectivity over a range of providers and technologies. In this environment of multiple providers and access media, NOMAD ${ }^{2}$ aims to provide a middleware that allows the

${ }^{1}$ European IST project IST-2000-26418: "Definition of new Services in the Area of $\underline{\text { Mobile }}$ Communication Network" - Next Generation Network Initiative

${ }^{2}$ European IST project IST-2001-33292: “Integrated Networks for Seamless and Transparent Service Discovery" 
integration of IP network technologies into a single platform capable of supporting user roaming between them.

However, as the original Internet Protocol design did not include such considerations, any of the above-described cases would cause an interruption of all active communications. A solution to the problem of Internet Mobility Management is provided through Mobile IP. For the issue of switching between devices while communicating, Mobile IP (in either IPv4 or IPv6 context) may be used to maintain a single point of attachment to the Internet, after a switch between access devices has been effected.

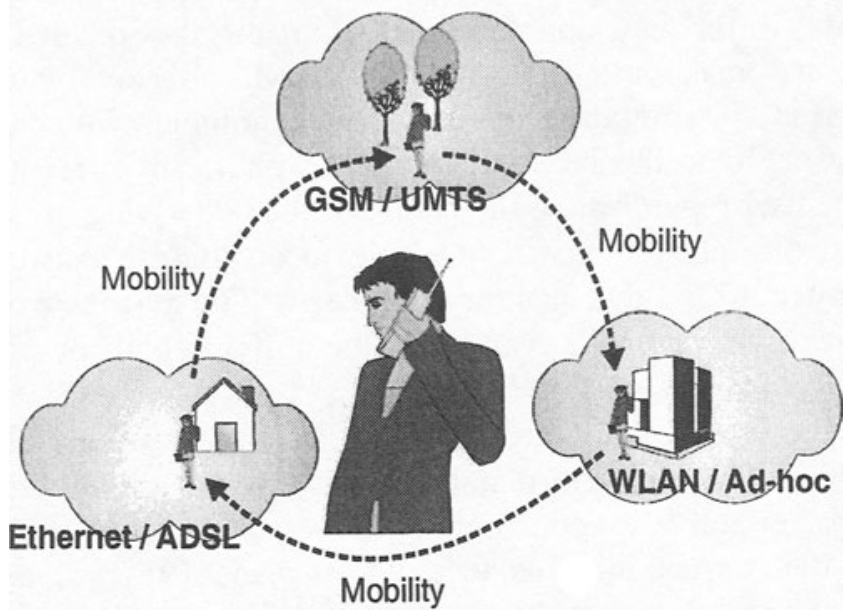

Figure 1: Integrated Network Platform

Within the above context, NOMAD aims at:

- Development of a transparent solution in the form of middleware based on IP mobility and the upcoming IETF standard for ad-hoc network support that will enable the realisation of an integrated network platform which will incorporate every available wireless (e.g. GSM, GPRS, UMTS, IEEE802.11a/b, HiperLAN2, et.) and wire-line access technology that is able to support Internet services. The usage of industry standards is imperative to ensure highest compatibility and industry acceptance.

- Location Information assisted Mobile IP handovers: Currently, Mobile IP handovers are managed by mechanisms (movement detection methods) [2] that are based either on the evaluation of the received wireless signal quality, or on periodic advertisements (beacons) that are broadcasted by Mobile IP mobility agents. Each of these mechanisms is associated with a given period of network service disruption. Within the boundaries of NOMAD, that assumes the existence of location information, alternative 
Mobile IP handover mechanisms will be studied that involve assistance from location information.

\section{TRANSPARENT SERVICE DISCOVERY AND PROVISION}

Integrated networks as a conglomeration of a range of heterogeneous access networks (terrestrial \& satellite, mobile \& fixed, wireless \& wire-line, symmetric \& asymmetric, public \& private) have certain characteristics that, on one hand offer new opportunities, but on the other impose new restrictions and make use of established service discovery technologies. Such platforms are composed of multiple technologies with very different basic features. Given the fact that integrated platforms present a seamless facade to the user regardless of the nature of the underlying layers, it is easy to assume that applications will not have to distinguish between different networks any more and that arbitrary services will be reachable ubiquitously. This however is not the case even under the unifying blanket of the Internet Protocol.

A major issue with integrated network platforms is in fact, that different segments of such a platform that happen to operate on different network technologies effectively constituting different Internet administrative domains that are termed into this document as planes [9]. Two users standing in the same room accessing the network via different planes may need to traverse vast ranges of Internet fabric in order to reach each other. That is, two neighbouring points in the physical world can be as removed from one another as possible in the Internet. This effectively demonstrates the discrepancy between the virtual world of the Internet and the physical world. A major requirement of service discovery on integrated network platforms is therefore the transparent bridging of the gap created by the discrepancy between locations in the physical world and locations in the virtual world of the network. 


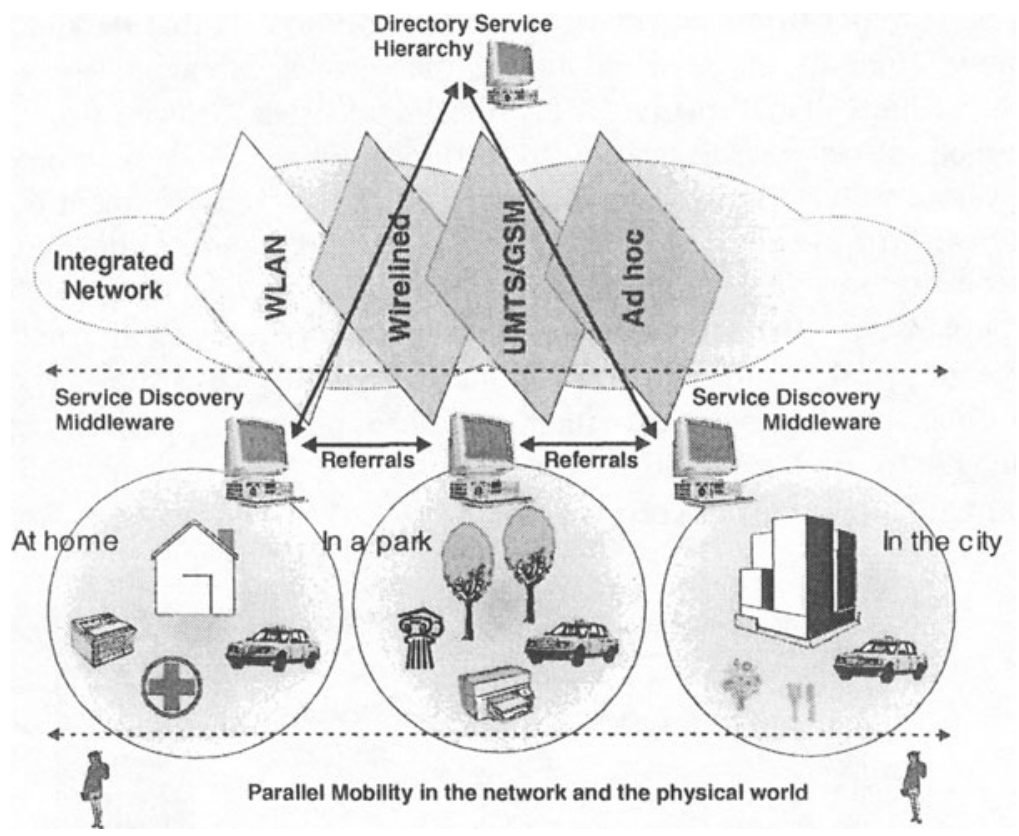

Figure 2: Different Planes of Integrated Networks

The implementation of a solution capable of realistically achieving widespread acceptance depends on the utilisation of widely accepted standards as building blocks. Internet standards in this area capable of providing mechanisms relevant to service discovery include the Service Location Protocol (SLP)[3] (see Figure 3), the Lightweight Directory Access Protocol (LDAP)[4] and the Dynamic Host Configuration Protocol (DHCP)[5] all of which operate on different layers. These protocols cover basic requirements of service discovery such as a means to specify the required service and formulate queries as well as ways for locating and communicating with the appropriate service provider. Service discovery functionality as specified by these protocols however focuses on Intranet resource discovery.

The actual discovery of the requested services is accomplished in two separate ways, either by directly contacting a known address that can supply the client with information on the available services (LDAP), or by broadcast. Broadcasts can be either focused on the local network (DHCP), or use mechanisms like multicast (SLP) to reach a much larger group of service agents without the need for predefined addresses. The later is a solution with clear advantages in a scenario whereby mobile clients constantly move from one physical location and segment of the integrated platform to another, thus also varying their access point within the integrated network platform and thus constantly in need of updating their information on the available 
services. A major disadvantage of broadcast solutions is that they can cause enormous amounts of overhead traffic that grows exponentially with the number of hops (Time-to-Live, TTL) the broadcast is allowed to make (i.e. the number of networks it is allowed to flood). Furthermore such broadcasts are necessary every time the mobile node changes its environment (i.e. after a handover), or in some cases every time a certain service is desired. Small TTLs on the other hand reduce the amount of signalling traffic generated, but coupled with the aforementioned discrepancy between the networked and the physical world, can lead to ineffective service discovery queries. Even clients and service providers in close physical proximity are not guaranteed to find each other, due to possibly large "virtual" distances separating them in the networked world.

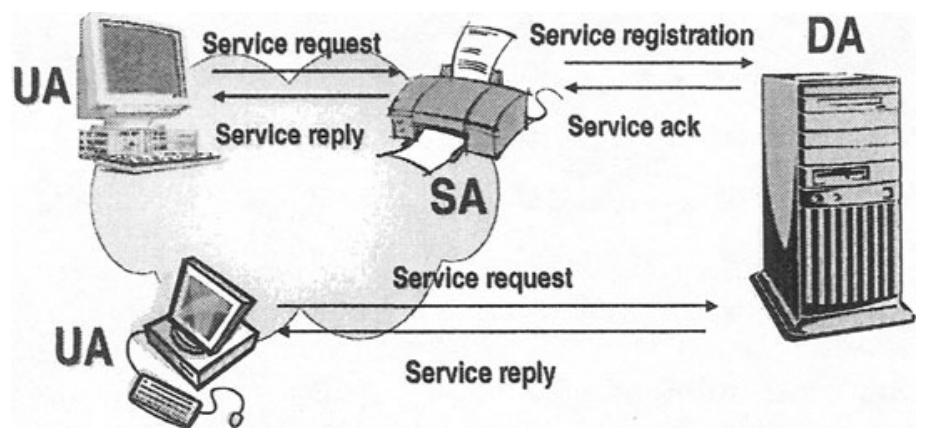

Figure 3: User Agents (UA), Service Agents (SA) \& Directory Agents (DA)

Another important issue regarding service discovery is the query mechanism used to extract the desired service from the set of available services in the network. Server based solutions like LDAP offer a rich and flexible query language that can be used to formulate very complicated and elaborate requests [6]. Last but not least, a solution implementing transparent service discovery must give the users the ability to protect themselves from push-type unsolicited service offers or requests.

A number of technical issues will be addressed before integrated networks and services can be provided. The following functions are to be considered:

- Investigate and facilitate the integration of Directory Agents and Directory Servers into gateways that can communicate with customers as well as Service Agents on one hand, and will allow for transparent service discovery between the different parts of the integrated network platform - through the use of Directory Service Referrals - on the other. Also investigate the usage of DHCP as a substitute for SLP when searching for a local Directory Server. 
- Investigate ways to extend and improve Referrals for usage in an integrated network platform. Directory Servers acting as Service Discovery middleware, that receive requests from a specific physical location and are connected to one or more networks could use an enhanced Referral mechanism to communicate with other "local" Directory Servers that serve a different network or physical area to exchange Information on queries and available services. This solution could help bridge the gap between different physical locations and parts of the integrated network.

\section{SERVICE INTEGRATION CONCEPT}

SMONET-NGNI is currently experiencing an era of convergence where growing numbers of services are increasingly being made available across fewer numbers of delivery devices. The logical outcome of this trend is that eventually most (popular) services will be available through one delivery device. However, we will consider a point further in the future where the services available on the converged device will no longer exist as monolithic, discrete and complete services. Such services can be thought of as compound services, where each compound service is constructed out of atomic services. Many of these services replicate functionality that other monolithic services already implement for their own purposes. In an era of ambient computing, where all services are available for hire at any location, it makes no sense for the replication of services at the atomic level: employing the $\mathrm{OO}$ paradigm as a metaphor, we are considering service reuse. A compound service can be "simulated" through the ad-hoc composition of atomic services into composite services. We propose a deployment model where services available in a network exist of atomic service components and that the software, hosted on the service convergence devices that exploit these services, compose the compound services for themselves when required. Such services have a lifetime that is dependent upon their current usefulness. We further propose that the software, hosted on the service convergence devices that compose these ad-hoc services will need to be persistent, act autonomously and display intelligent characteristics in order to function in an environment where domain knowledge is, by definition, always incomplete. Software complying with these requirements already exists in the form of Intelligent Agent technology. FIPA-OS is and intelligent agent development toolkit that implements the specifications of the main international agent standards Body: FIPA ${ }^{3}$. We develop a notional

${ }^{3}$ Foundation for Intelligent Physical Agents: http://www.fipa.org/ 
design in this document that exploits and extends the functionality of FIPA$\mathrm{OS}$ as the mechanism to deliver an implementation of the ad-hoc service discovery and composition mechanism.

It is unreasonable to expect that a user could remember how many compound services there are on a network (or where they exist) or to compose composite services from any atomic services available. Therefore, this deployment model requires that the agents consuming the atomic services encapsulate not only a knowledge of the compound services that a user is likely to request and the atomic services extant in the network but also of the process(es) involved in decomposing atomic service descriptions from a compound service request and executing a composite service to correctly meet the requirements of the original compound service request.

However, we judge that it is likely that the most frequently requested services will actually exist as instances of compound services to ensure that a balance between service reuse and host resource consumption are adequately managed. We also judge that the devices supporting client agent deployment will always place demands upon the agent that restrict its footprint, indirectly limiting the client agent's degree of sophistication. In such an environment a possible set of interactions, at the service composition level, are described below:

1. Agents offering atomic services register service descriptions with a middle agent acting as a directory facilitator (DF) [10].

2. A client agent contacts the DF, making a simple request for the location of a compound service on behalf of a user

3. The middle agent acting as DF conducts local and remote searches for an instance of the compound service. If no compound services exist, the DF employs its understanding of service composition to reason over candidate atomic service components that could be employed in the construction of a bespoke composite service.

4. The middle agent acting as DF conducts local and remote searches for instances of the decomposed candidate atomic services.

5. The middle agent acting as DF reasons over how best to combine the candidate atomic services into an appropriate composite service.

6. The middle agent acting as DF reasons over the ability of the client agent to assemble and execute the atomic services in the appropriate manner. If the client agent is determined to not possess the required sophistication, the services of additional middle agents will be obtained.

7. The middle agent acting as DF will be subscribed to a middle agent acting as a mediation agent (MeA) which shares part of the DF's service composition knowledge domain but also has knowledge of process models that effectively determine the correct invocation sequences and 
relationships between the atomic service components that will deliver the required composite service.

8. The middle agent acting as DF notifies the middle agent acting as MeA of the requirement to execute a process model in support of the target composite service employing a list of atomic services that meets the specification of the originally requested compound service.

9. The middle agent acting as DF informs the client agent of the service description (includes the agent $\mathrm{ID}$ and its address) of the middle agent acting as MeA.

10. The client agent contacts the MeA, making a simple request for a compound service as if it were communicating with an agent specialising in that compound service.

11. The middle agent acting as $\mathrm{MeA}$ constructs a composite service execution plan from its knowledge of the target compound service process model and makes the appropriate requests to the atomic service providing agents.

12. The middle agent acting as MeA informs the client agent of the result of the composite service's execution and also passes any supporting information back to the client agent.

\section{DYNAMIC PLATFORM DISCOVERY}

SMONET-NGNI examined dynamic platform discovery with entity discovery. Entity discovery means the process of the agent or the platform on the mobile device being able to detect the network to locate local services. Local in this case means the local network the user has roamed into, like the hotel network that might not necessarily be connected to the Internet, or might have an extra charge for doing so.

Before locating agents on the network, there needs to be a mechanism that allows the agents to detect when the network is present. There are few possible ways of doing this. Have an agent on the platform whose (only) functionality is to monitor the network. FIPA has a concept of such an agent called Monitor Agent (MA) in the Nomadic Application Support specification. FIPA MA will

- Observe the quality of service of MTPs ${ }^{4}$ and $\mathrm{MTCs}^{5}$, see 0

- Measure (if there are no other means to obtain the required information) the quality of service of an MTP and MTC

- Collect information from the observing and measuring sources

- Analyse the information

\footnotetext{
${ }^{4}$ Message Transport Protocol

${ }^{5}$ Message Transport Connection
} 
In this case an additional functionality would be required, that is other agents should be able to subscribe to the MA to receive information it has detected, i.e. when the network is present and when not.

Java can be used hardware independently trough a Java Virtual Machine be used to detect the network presence. Although Java sockets don't directly offer help with detecting the network, it's easy to take advantage of Java's blocking $\mathrm{I} / \mathrm{O}$ (i.e. the fact that if read or write stalls if $\mathrm{I} / \mathrm{O}$ device is not ready). By creating a helper class that allows timeouts on socket connection stalling can be avoided. We can then check whether network is present - if the entity can't create a connection to a socket, the network is not present.

After the network connection has been established the platform needs to locate services in the network. This is done easily by first locating a local $\mathrm{DF}$, and then asking it for local services available. First though, the platform on the mobile device needs to locate the local platform(s). This can be done with IP multicasting to a known address, and if time-to-live ${ }^{6}$ (TTL) is kept small, the multicast will stay local. With a multicast transport service, a single node can send data to many destinations by making just a single call on the transport service ${ }^{7}$ - this obviously will not only decrease the network load, but also there isn't need to know about all potential platforms that might host desired services.

Because of the nature of roaming from one network to another (or loosing the network altogether), normal FIPA-defined DF cross registration can't be used since the DFs can't deregister once the connection is lost. This would cause problems when roaming into a new network while doing federated DF searches. It is because of this that it is also assumed the mobile DF doesn't have prior knowledge about any other DFs on the network - in fact for simplicity's sake it's suggested that the DF doesn't have a persistent database.

Once the remote platform is known, the mobile platform's DF can register the remote platform's $\mathrm{DF}^{8}$ in its database. After that the services can be searched by the agents on the mobile platform without knowledge of any remote platforms. [13]

${ }^{6}$ A counter that is decremented every time the packet passes through a hop i.e. a router between the networks.

${ }^{7}$ Java offers tools for IP multicasting in java.net package - and because of Java's way of abstracting underlying technology it is hardware independent.

${ }^{8}$ FIPA states that if DFs exist on a platform, at least one of them needs to be called by the default DF name (df@<platform-name $>$ ). 


\section{CONCLUSIONS}

The results of current developments in both wireless data communications and mobile computers are being combined to facilitate a new trend: nomadic computing. Compared to today's traditional distributed systems, the nomadic computing environment is very different in many respects. Bandwidth, latency, delay, error rate, quality of display and other non-functional parameters may change dramatically when a nomadic enduser moves from one location to another and thus from one computing environment to another, for example, from a wire-line LAN to a UMTS network.

Confronted with these circumstances, the nomadic end-user would benefit from having the following functionality provided by the infrastructure: information about expected performance, agent monitoring and controlling the transfer operations, and adaptability. It is in situations like these where agent technologies will become very useful. User agents on user's device can discover the network, and locate and communicate with services on the network transparently to the user.

SMONET-NGNI use cases and demonstrators illustrate a scenario of adhoc service discovery. SMONET-NGNI furthermore described how ad-hoc networking can be solved using a FIPA agent platform, especially a design and implementation for ad-hoc networking for FIPA-OS. Meta-services have been developed which enable other application services to be discovered (through incomplete, and heterogeneous, network connectivity environments) and composed from service elements.

On the other hand the NOMAD project deals with the integration of location aware service discovery mechanisms, handover procedures and service / user profiling, by developing technology that allows users to freely roam across existing and future network infrastructures. We analyse the NOMAD Network Platform concept, the methods for transparent discovery and seamless provision of services and the integration of service / user profiles. The NOMAD platform will be available for testing and refining the implemented software during the project lifetime. The overall target of NOMAD is to derive products enabling the integration of existing wire-line and wireless technologies with seamless connectivity and intelligent service discovery provision, increasing user satisfaction and adding value to existing services. 


\section{ACKNOWLEDGEMENTS}

The project NGNI (IST-2000-26418) and NOMAD (IST-2001-33292) are funded by the European Commission as IST projects. The authors wish to acknowledge the European Commission for their support. The authors also wish to acknowledge their gratitude and appreciation to all NGNISMONET and NOMAD partners for their strong support and valuable contribution during the various activities presented in this paper.

\section{REFERENCES}

[1] Mohr, W., "Alternative Vorschläge zur Spektrumsnutzung für IMT-2000/UMTS", Spektrumsworkshop ITU-R, October 2000, Geneva, Switzerland

[2] Fikouras NA and Görg C, (2000). Performance Comparison of Hinted and Advertisement Based Movement Detection Methods for Mobile IP Hand-offs, 7th European Conference on Fixed Radio Systems and Networks. Dresden, Germany

[3] Guttman, E., Perkins C., Veizades J. and Day M., "Service Location Protocol, Version 2", RFC 2608, June 1999

[4] M. Wahl, T. Howes, S. Kille, "Lightweight Directory Access Protocol (v3)" RFC2251-2256,2829-2831, December 1997

[5] Droms, R.,"Dynamic Host Configuration Protocol” RFC 2131, March 1997

[6] Howes, T., "The String Representation of LDAP Search Filters" RFC 2254, December 1997

[7] The W3 Consortium, "Extensible Markup Language (XML)", WWW-site (16.4.2001) http://www.w3.org/XML

[8] Drane, C. (1998) "Positioning Systems in Intelligent Transportation Systems", Artech House, Boston, London

[9] I. Fikouras, Peer-to-Peer Service Engineering for Integrated Networks, in: Elizabeth Cooper (eds.) World Markets Series - Business Briefing - Wireless Technology 2002, WMRC PLC.

[10] FIPA Agent Management Specification, available at http://www.fipa.org/specs/ fipa00023/

[11] FIPA Nomadic Application Support Specification, available at http://www.fipa.org/ specs/fipa00014l

[12] Ad-hoc FIPA Work Plan (F-IN-00027) by Michael Berger and Michael Watzke, Siemens AG 2001

[13] SMONET-NGNI: Deliverable 04; Specification of Service Discovery Platform Architecture; IST project of NGNI (IST-2000-26418) 